\title{
Determination of multidrug resistance mechanisms in Clostridium perfringens type $A$ isolates using RNA sequencing and 2D-electrophoresis
}

\author{
Yu-Hua Ma ${ }^{1,2}$ and Gui-Sheng Ye $\mathrm{e}^{1,2}$ \\ ${ }^{1}$ State Key Laboratory of Plateau Ecology and Agriculture, Qinghai University, Xining, China \\ ${ }^{2}$ College of Agriculture and Animal Husbandry, Qinghai University, Xining, China
}

\begin{abstract}
In this study, we screened differentially expressed genes in a multidrug-resistant isolate strain of Clostridium perfringens by RNA sequencing. We also separated and identified differentially expressed proteins (DEPs) in the isolate strain by two-dimensional electrophoresis (2-DE) and mass spectrometry (MS). The RNA sequencing results showed that, compared with the control strain, 1128 genes were differentially expressed in the isolate strain, and these included 227 up-regulated genes and 901 down-regulated genes. Bioinformatics analysis identified the following genes and gene categories that are potentially involved in multidrug resistance (MDR) in the isolate strain: drug transport, drug response, hydrolase activity, transmembrane transporter, transferase activity, amidase transmembrane transporter, efflux transmembrane transporter, bacterial chemotaxis, ABC transporter, and others. The results of the 2-DE showed that 70 proteins were differentially expressed in the isolate strain, 45 of which were up-regulated and 25 down-regulated. Twenty-seven DEPs were identified by MS and these included the following protein categories: ribosome, antimicrobial peptide resistance, and ABC transporter, all of which may be involved in MDR in the isolate strain of $C$. perfringens. The results provide reference data for further investigations on the drug resistant molecular mechanisms of $C$. perfringens.
\end{abstract}

Key words: Clostridium perfringens; Multidrug resistance; RNA sequencing; 2D-electrophoresis; Molecular mechanism

\section{Introduction}

Clostridium perfringens, an important zoonotic pathogen, is capable of causing necrotic enteritis and food poisoning in humans $(1,2)$. Bacterial drug resistance can occur through inherent gene mutations and foreign gene acquisition (3). With inherent gene mutation acquisition, the resistance gene exists in the bacterial genome, and the drug resistance is typically species-specific, such as penicillin resistance in Pneumococcus (4). When bacteria develop drug resistance through the acquisition of foreign genes, the resistance gene may be located in the bacterial genome, or in a plasmid, transposon, or integron; hence, resistance genes can be spread via plasmids, transposons and integrons among the various carriers, making bacterial drug-resistance patterns more complex and diverse. The inactivating or modifying enzymes produced by bacteria mainly cause a loss of biological activity in an antibiotic, and this loss involves bacterial $\beta$-lactam-inactivating enzymes, aminoglycoside-modifying enzymes, and chloramphenicol acetyltransferases (5).

Under antibiotic pressure, an alteration of the target bacterial protein can occur in the drug-binding site of the intracellular membrane and this reduces the affinity of the drug for its target, thereby eliminating the efficacy of the antibiotic. This is a common mechanism of drug resistance in bacteria (6). The efflux pump is another primary cause of bacteria resistance to many drugs (7), such as ATP-binding cassette, ABC transporter, and drug-resistant nodulation division family (8). Due to the efflux pump, Escherichia coli, Staphylococcus aureus, etc. have multiple resistance to tetracycline, fluoroquinolones, and $\beta$-lactam among others (9).

The $C$. perfringens $\operatorname{Tet} A(P)$ protein is an endometrial protein that regulates tetracycline active efflux. It consists of 420 amino acids and 12 transmembrane domains (10). The resistance mode by reducing membrane permeability is relatively rare in gram-positive bacteria, but vancomycinresistant Staphylococcus aureus can specifically modify the cell wall to reduce permeability, thereby reducing the amount of drug entering the cell (11). Streptococcus pneumoniae can produce $V n c R-V n c S$ and other cell wall regulators to change the cell wall permeability and develop resistance (12). 
Bacterial biofilm is an important cause of bacterial resistance (13); it can reduce the penetration of antibacterials due to the barrier function of extracellular polysaccharides (14). The growth of bacteria in biofilm is slow and the sensitivity to antibiotics is reduced (15). The induced expression of rpoS gene in the biofilm formation stage of Escherichia coli may be caused by the formation of drug-resistant subgroups in the deep layer of the mature biofilm (16). C. perfringens can form biofilms, and type IV pilus and C CPA protein are necessary for biofilm formation. The biofilm from $C$. perfringens resists oxygen and antibiotics effectively (17).

In this study, we analyzed differentially expressed genes (DEGs) and differentially expressed proteins (DEPs) in a multidrug resistance (MDR) isolate of type A C. perfingens. The study used RNA sequencing (RNA-Seq), two-dimensional electrophoresis (2-DE), and mass spectrometry (MS) to investigate the transcriptome and proteome of the MDR isolate and a control strain of $C$. perfringens.

\section{Material and Methods}

\section{Strains}

An MDR strain of $C$. perfringens type A was isolated, identified, and preserved by the Laboratory of Animal Disease based at the Qinghai-Tibet Plateau in the Department of Veterinary Medicine, College of Agriculture and Animal Husbandry, Qinghai University, China (18). The standard C. perfringens type A strain, CICC22949, purchased from the China Center of Industrial Culture Collection, was used as the control strain. In the preliminary experiments on the isolate strain of $C$. perfringens, we found that the minimum inhibitory concentrations of kanamycin sulfate, minocycline hydrochloride, clindamycin hydrochloride, doxycycline hydrochloride, and novobiocin were higher than those of the control strain.

\section{Total RNA extraction, cDNA library construction, and} sequencing

C. perfringens were grown overnight at $37^{\circ} \mathrm{C}$ in liquid medium of sulfate glycolate after sterilization. The cells were harvested by centrifugation at $10,625 \mathrm{~g}$ for $3 \mathrm{~min}$ at room temperature when $C$. perfringens were grown with an initial OD600 of 0.6. Total RNA of the C. perfringens isolate strain and control strain were extracted using the RNA Isolater total RNA extraction reagent (Cat\#401, Vazyme, China) according the manufacturer's instructions. An RNA integrity number was determined using an Agilent 2100 bioanalyzer (Agilent Technologies, USA). After quantification, $10 \mu \mathrm{g}$ of the extracted RNA was digested by DNase I at $37^{\circ} \mathrm{C}$ for $30 \mathrm{~min}$. Ribosomal RNA was removed using a Ribo-Zero ${ }^{\mathrm{TM}}$ magnetic kit (Epicentre, USA). The cDNA library was constructed using the NEB Next $^{\mathbb{R}} \quad$ UltraTM directional RNA library prep kit from Illumina (NEB, USA). Random primers and first strand synthesis reaction buffer (NEB) were added to the mRNA solution to allow cDNA synthesis to occur. Following purification, end repair and joint connection were conducted to give 300-500 bp ligated cDNA molecules. After polymerase chain reaction (PCR) amplification and library construction, sequencing was performed using Illumina Hiseq $^{\text {TM }} 2500$.

\section{Genome comparison and DEG analysis}

The raw sequencing reads were filtered for quality control to obtain clean reads. These reads were then mapped to the reference genome using SOAP2 (19). The distribution and coverage of the reads on the reference sequence were analyzed. DEGs were screened by analysis of the significance of digital gene expression profiles (20), followed by enrichment analysis of gene ontology (GO) terms by GO TermFinder software (http://smd.stanford. edu/help/GO-TermFinder/GO_TermFinder_help.shtml) and KEGG pathways (21).

\section{Two-dimensional electrophoresis}

Culture sample $(1 \mathrm{~g})$ was decanted and $1 \mathrm{~mL}$ of lysis buffer (9 mol/L UREA, 4\% CHAPS, 1\% IPG buffer, 1\% DTT was added. The sample was disrupted by ultrasonication $(80-100 \mathrm{~W}, 3 \mathrm{~min})$ and centrifuged $(10,625 \mathrm{~g}$ for $30 \mathrm{~min}$ at $4^{\circ} \mathrm{C}$ ) to remove the precipitate. Next, $1 \mathrm{~mL}$ of pre-cooled acetone was added and the sample was kept at $-20^{\circ} \mathrm{C}$ overnight. The supernatant was removed after centrifugation $\left(10,625 \mathrm{~g}\right.$ for $30 \mathrm{~min}$ at $\left.4^{\circ} \mathrm{C}\right)$. The precipitate was dried and $500 \mu \mathrm{L}$ of protein hydration solution was added. The extracted protein was quantified and used for 2-DE. Briefly, $150 \mu \mathrm{g}$ of the protein sample was removed, dry strips were prepared ( $\mathrm{pH} \mathrm{3-10} \mathrm{NL} \mathrm{IPG),} \mathrm{and} \mathrm{run} \mathrm{for} \mathrm{the}$ first-dimension isoelectric focusing. The equilibrated strips were placed in the gel slab for the second-dimensional sodium dodecyl sulfate-polyacrylamide gel electrophoresis. After electrophoresis, the gel was stained with Coomassie blue. Following decolorization, the gel was scanned by ImageScanner (GE Healthcare, USA).

\section{MS detection and DEP analysis}

Selected granules were excised from the gel and transferred to $1.5-\mathrm{mL}$ tubes for decolorization. The sample was digested with trypsin and $100 \mu \mathrm{L}$ of $60 \%$ acetonitrile (ACN); $0.1 \%$ trifluoroacetic acid (TFA) was added. The mixture was ultrasonicated for $15 \mathrm{~min}$ and then lyophilized. After lyophilization, $2 \mathrm{~mL}$ of the digested sample was collected and $20 \%$ ACN was added. A $1-\mathrm{mL}$ aliquot of the sample was spotted onto the sample target and $0.5 \mu \mathrm{L}$ of supersaturated $\mathrm{CHA}$ solution was spotted onto the corresponding target. The sample was air dried and the sample target was blown with nitrogen gas before being placed into the target slot for the MS analysis. The laser source was $\mathrm{Nd}$ :YAG laser with $355 \mathrm{~nm}$ wavelength, and the peptide mass fingerprinting mass scan range was 800-4000 Da. Parent ions with signal-to-noise ratios greater than 50 were selected for tandem MS (MS/MS) analysis. 


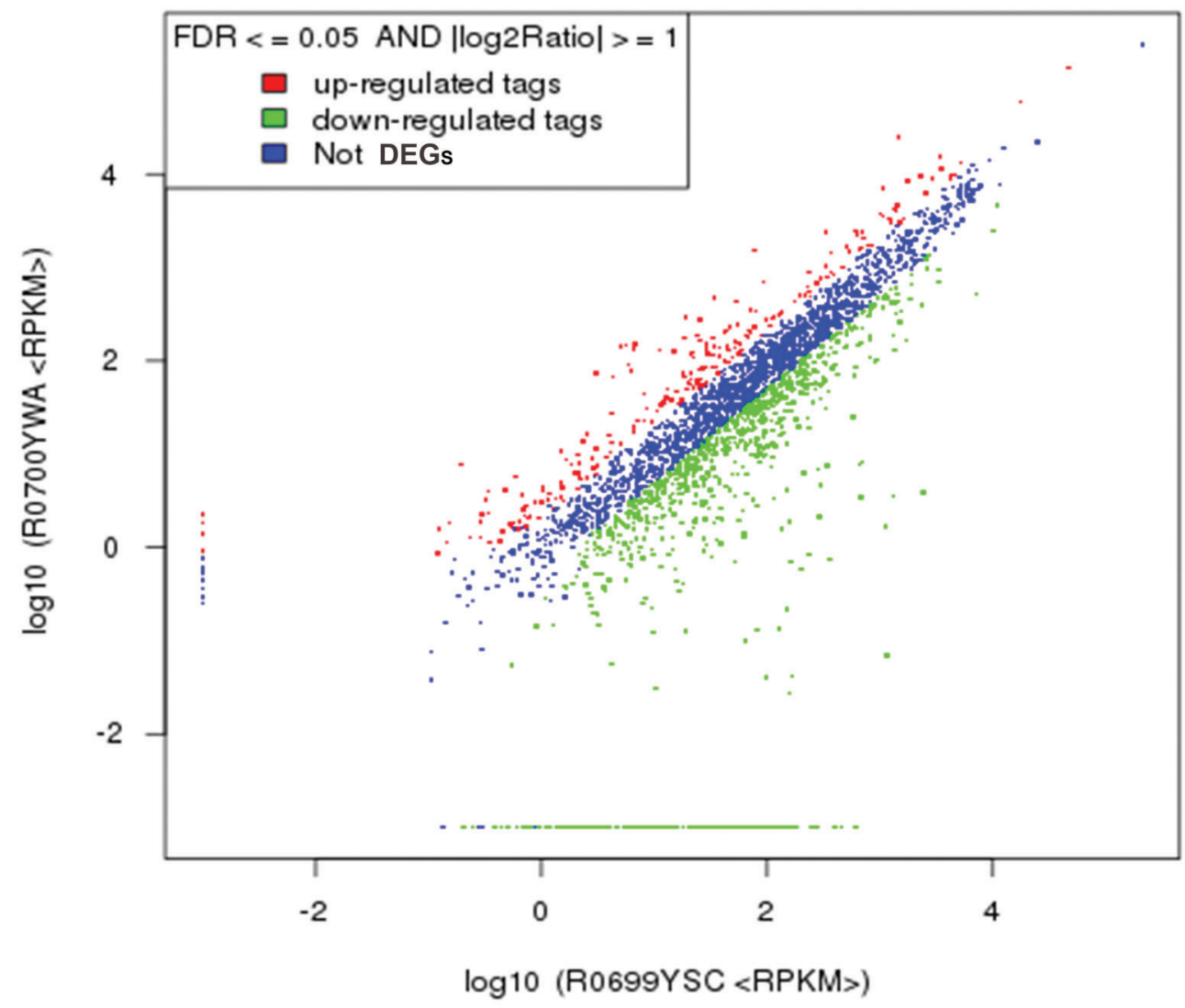

Figure 1. Differentially expressed genes (DEGs) in the multidrug resistance (MDR) isolate strain of $C$. perfringens. The red color represents up-regulated tags, the green color represents down-regulated tags (fold change) and the blue color represents no significant DEGs. FDR: false discovery rate.

The MS/MS was performed with a laser excitation of 2500 times and $2 \mathrm{kV}$ of collision energy, and with the collisioninduced decomposition shut down. The MS data were analyzed using Mascot (SCIEX, USA). GO and Kyoto Encyclopedia of Genes and Genomes (KEGG) analyses (http://www.kegg.jp/) of the DEPs were conducted.

\section{Results}

\section{Transcriptome sequencing data and DEG analysis}

A total of $28,563,164$ reads were obtained from the MDR isolate strain of $C$. perfringens by transcriptome sequencing. Specifically, $83.97 \%$ of the reads were mapped to the $C$. perfringens genome, $60.71 \%$ were mapped to $C$. perfringens genes, and the unique matches reached $83.35 \%$. Concurrently, 26,254,552 reads were obtained from the $C$. perfringens control strain by transcriptome sequencing. Specifically, $89.41 \%$ of the reads were mapped to the $C$. perfringens genome, $72.6 \%$ mapped to $C$. perfringens genes, and the unique matches reached $88.65 \%$.

For the MDR isolate strain of $C$. perfringens, the vast majority of gene coverages were higher than $10 \%$; this included 2000 gene coverages between 90 and 100\%. For the control strain, all gene coverages were higher than $10 \%$; this included 2437 gene coverages between 90 and $100 \%$.

A total of 1128 DEGs (FDR $\leqslant 0.05$ and |log2Ratio $\mid \geqslant 1$ ), including 227 up-regulated genes and 901 down-regulated genes, were screened in the MDR isolate strain relative to the control strain (Figure 1).

We performed GO and KEGG enrichment analyses on 1128 DEGs in the MDR isolate strain of $C$. perfringens. The results showed that these DEGs participated in 648 biological processes (Figure S1A) wherein defense responses, drug transport, drug responses, and lactamase transport may be related to multidrug resistance in the MDR isolate strain of $C$. perfringens. These DEGs are derived from 80 cellular components (Figure S1B) wherein the $A B C$ transporter, ATP-dependent transmembrane transporter, transmembrane transporter, protein membrane complex, and ribosome may be related to multidrug resistance in the MDR isolate strain of $C$. perfringens. Moreover, these DEGs have 399 molecular functions (Figure S1B) wherein hydrolase, transport protein, transmembrane transporter activity, 


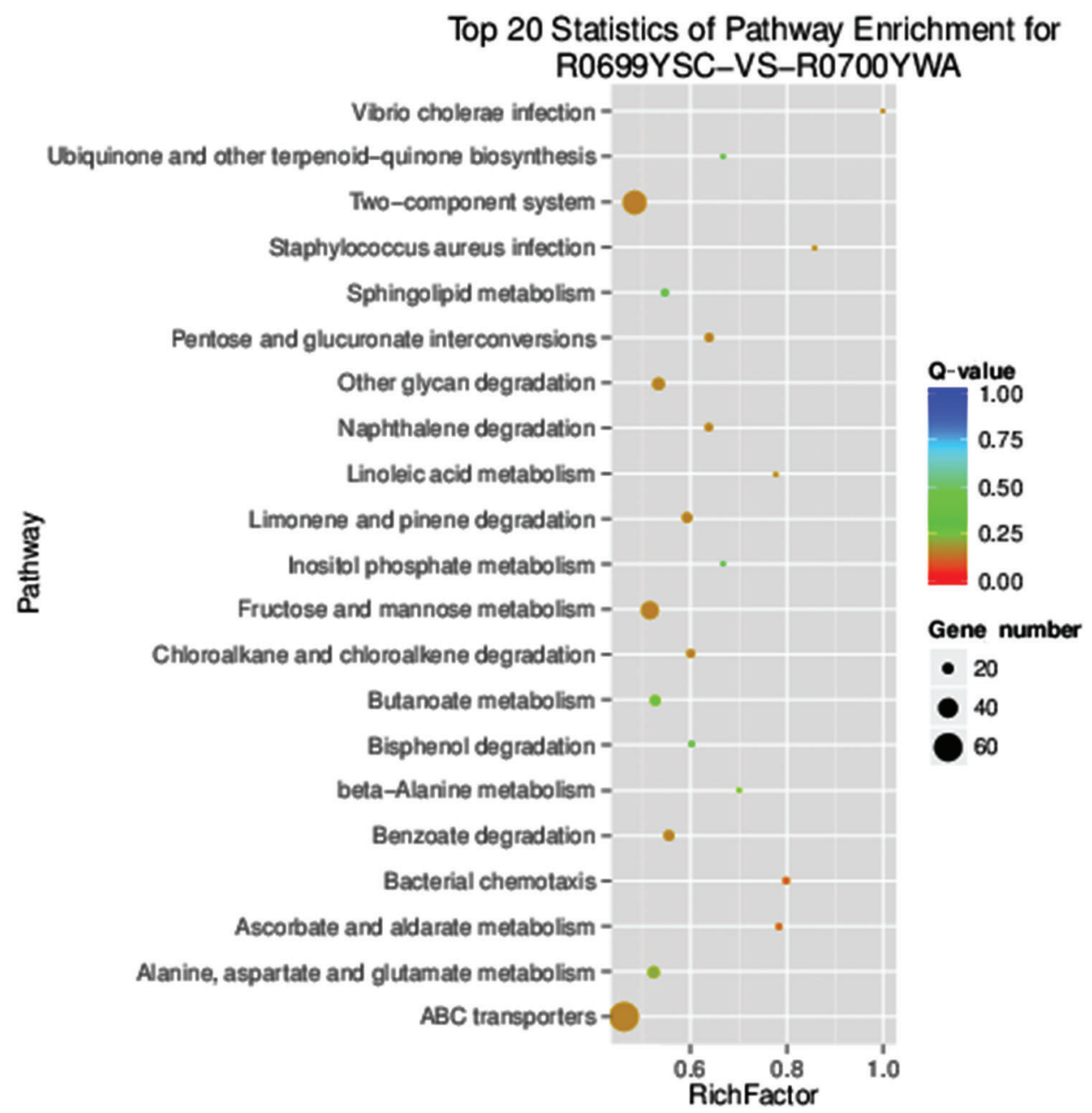

Figure 2. KEGG enrichment pathway of differentially expressed genes (DEGs) in the multidrug resistance isolate strain of $C$. perfringens. The top 20 enriched pathways are shown in the graph, different color means different $Q$-value, and the size of the bubble represents the number of DEGs.

transferase activity, amidase transmembrane transporter, transcription factor activity, and efflux transmembrane transporter activity may be related to multidrug resistance in the MDR isolate strain of $C$. perfringens.

The 1128 DEGs are involved in 122 KEGG pathways (Figure 2) wherein bacterial chemotaxis, ABC transporter, and $\beta$-lactam resistance may be associated with multidrug resistance in the MDR isolate strain of $C$. perfringens.

\section{2-DE, MS, and DEP analyses}

The 2-DE results (Figure 3 ) showed clear protein spots for the MDR isolate strain of $C$. perfringens and the control strain. The trend of the proteins was consistent within each group, with good reproducibility. Next, an image analysis was conducted using ImageScanner and PDquest 8.0 (Bio-Rad, USA) software, and the DEPs were screened using the following criteria: fold changes $>2$ or $<0.5$ for the analytical values, and $\mathrm{P}$-values $<0.05$ by the $t$-test. $A$ total of 70 DEPs, 45 of which were up-regulated and 25 were down-regulated, were identified in the isolate strain relative to the control strain.

Twenty-seven DEP spots with large fold-changes for up-regulated expression were selected from the MDR isolate strain of $C$. perfringens for enzymatic hydrolysis and desalination in the gel. The digested samples were re-dissolved with ACN and spotted onto the sample target for Maldi-TOF/TOF analysis. The MS data were used for protein identification using Mascot search software. The results showed that the 27 protein spots were identified successfully.

GO and KEGG enrichment analyses were performed on the amino acid sequences of the 27 DEP spots 

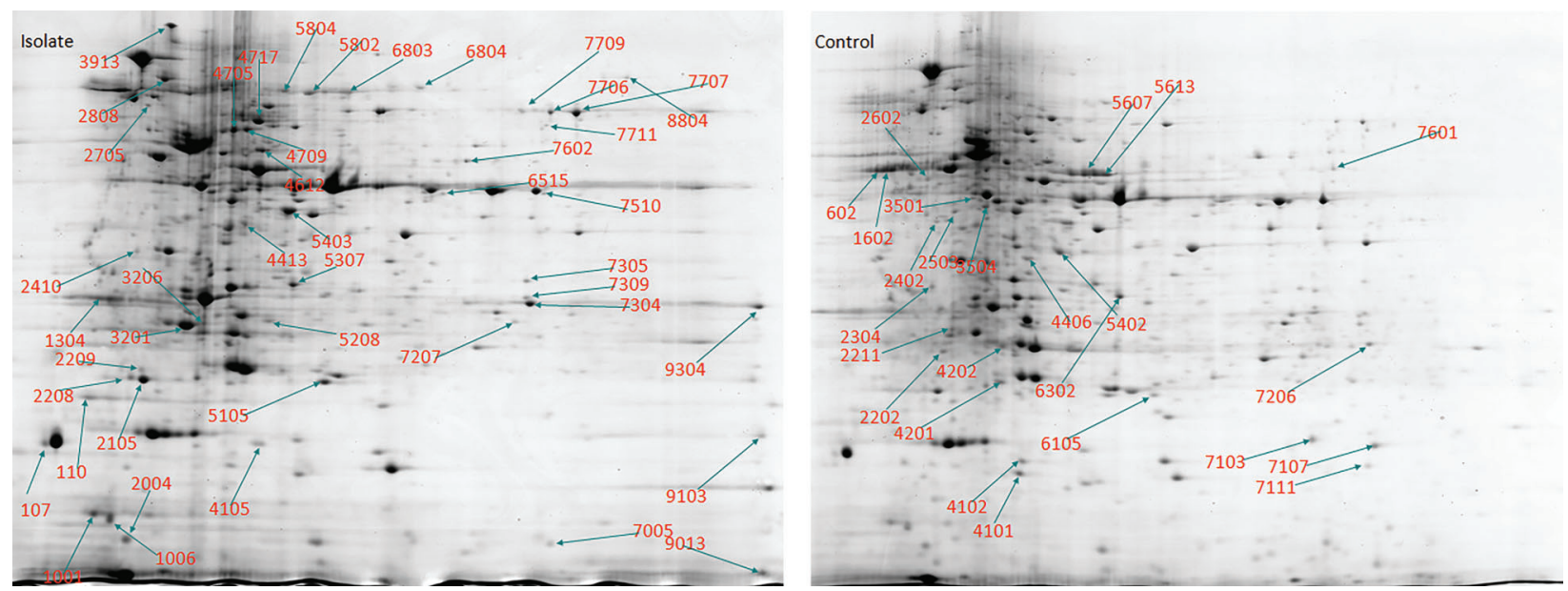

Figure 3. Comparative analysis of proteins of $C$. perfringens by 2D- electrophoresis. The image shows the differential expression of protein spots from the proteins extracted from (left) multidrug resistance isolate strain of $C$. perfringens, and (right) control strain. Proteins whose fold change were higher than 2 or less than 0.5 were selected for further analysis. The arrows refer to the differentially expressed protein spots.

Biosynthesis of secondary metabolites
Metabolic pathways
Glycolysis / Gluconeogenesis
Purine metabolism
Ribosome

Figure 4. KEGG pathway enrichment analysis of differentially expressed proteins in the multidrug resistance isolate strain of $C$. perfringens. Differentially expressed proteins were categorized according to their gene ontology terms and in each category the number of proteins and their P-values are shown in the graph. The X-axis shows the percentage of differentially enriched proteins.

successfully identified by MS. The results showed that the DEPs found in the MDR isolate strain of $C$. perfringens participated in 292 biological processes (Figure S2A), 111 of which were significantly enriched. These DEPs were related to 44 cellular components (Figure S2B), 18 of which were significantly enriched. Moreover, these DEPs were involved in 142 molecular functions (Figure S2C), 37 of which were significantly enriched.

The 27 DEPs participating in 24 KEGG pathways (Figure 4) include ribosomes, antibiotic biosynthesis, antimicrobial peptide resistance, and $A B C$ transporters. The ribosomal pathway, antimicrobial peptide resistance, and $A B C$ transporters may be related to multidrug resistance in the MDR isolate strain of $C$. perfringens.

\section{Discussion}

Bacteria will trigger a variety of mechanisms against drugs under the sustained pressure of antibiotics. $\beta$-lactamase is a primary cause of resistance to $\beta$-lactam antibiotics (22); its encoding gene can spread among bacteria by transformation, transduction, conjugation, and other ways, such as in ESBLs-producing bacteria (23). Aminoglycoside modifying enzymes can help bacteria to develop resistance to aminoglycoside antibiotics (24), as the encoding gene can transfer among bacteria through plasmid conjugation, and cause drug resistance (25). Bacteria can also develop drug resistance by increasing the number of target proteins (26). Resistance to $\beta$-lactam antibiotics can 
be caused by changing the number of penicillin binding proteins or deleting it. This kind of drug resistance is common in bacteria, which is dependent on $\beta$-lactam antibiotics rather than $\beta$-lactamases (27). The resistance of the bacteria to rifampin is due to the change in the beta subunit of the RNA polymerase in the bacteria, thus reducing the drug's binding capacity and developing resistance (28).

C. perfringens isolated from piglets in Thailand was reported to have an MDR phenotype (29). This bacterium has also been reported to be capable of inactivating antibiotics via production of drug-inactivating or drugmodifying enzymes (30). In chloramphenicol-resistant C. perfringens, the product encoded by the catP resistance gene, which is located on the Tn4453 transposon, can inactivate chloramphenicol and spread via plasmid conjugation (31). In lincomycin-resistant $C$. perfringens, the transposon-located tISCpe8 nucleotidyltransferase, which is encoded by the t/nuP resistance gene and spreads by plasmid conjugation, can inactivate lincomycin (32). Additionally, because of gene transfer, tet(M) resistance gene appeared in $C$. perfringens type $C$, carrying tetB resistance gene (33). C. perfringens can develop quinolone resistance by altering the sites of drug action in the genes encoding DNA gyrase and topoisomerase IV. Mutated gryA DNA gyrasegenein $C$. perfringens, and the mutant bacterium grown in an environment with gatifloxacin and ciprofloxacin showed a certain degree of resistance to these antibiotics (34). Additionally, C. perfringens acquired linezolid resistance via a new mutation in the highly conserved region of the $50 \mathrm{~S}$ ribosomal protein $\mathrm{L} 4$ gene, rpID (35). When a drug reaches a certain concentration in bacteria, the expression of proteins related to the active efflux system increases, thereby pumping the drug out of the cells. By transferring a putative coding gene of an $A B C$ transporter from a ciprofloxacin-resistant

\section{References}

1. Rood JI, Cole ST. Molecular genetics and pathogenesis of Clostridium perfringens. Microbiol Rev 1991; 55: 621-648.

2. Smerdon WJ, Adak GK, O'Brien SJ, Gillespie IA, Reacher $M$. General outbreaks of infectious intestinal disease linked with red meat, England and Wales, 1992-1999. Commun Dis Public Health 2001; 4: 259-267.

3. Hall BG. Predicting the evolution of antibiotic resistance genes. Nat Rev Microbiol 2004; 2: 430-435, doi: 10.1038/ nrmicro888.

4. Hanage WP, Huang SS, Lipsitch M, Bishop CJ, Godoy D, Pelton SI, et al. Diversity and antibiotic resistance among nonvaccine serotypes of Streptococcus pneumoniae carriage isolates in the post-heptavalent conjugate vaccine era. $J$ Infect Dis 2007; 195: 347-352, doi: 10.1086/510249.

5. Zorov SD, luzenkov lu V, Severinov KV. Low-molecular weight inhibitors of bacterial DNA-dependent RNA polymerase. Mol Bio I(Mosk) 2006; 40: 971-981.
C. perfringens strain into a wild-type strain, a study found that not only was the accumulation of ethidium bromide reduced in the recombinant strain, but also the accumulation of norfloxacin and ciprofloxacin was reduced in the cells (36).

In this study, we screened 1128 DEGs from a MDR isolate strain of $C$. perfringens using RNA-Seq. Bioinformatics analysis showed that these genes participated in biological pathways including drug transport, drug response, amidase transport, hydrolase activity, transferase activity, along with an amidase transmembrane transporter, efflux transmembrane transporter, bacterial chemotaxis, $A B C$ transporters, and a $\beta$-lactam resistance gene, all of which may be related to multidrug resistance in the isolate strain of $C$. perfringens type A. Furthermore, we obtained 70 DEP spots, including 45 that were up-regulated and 25 that were down-regulated in the MDR isolate strain of C. perfringens by $2-\mathrm{DE}$. Of these, 27 protein spots with relatively large fold-changes in up-regulated expression were identified by MS, and these proteins participate in various biological pathways. The proteins in these spots, which are potentially related to multidrug resistance in the MDR isolate strain of $C$. perfringens, include ribosomes, antimicrobial peptide resistance determinants, and $A B C$ transporters.

\section{Supplementary Material}

Click here to view [pdf]

\section{Acknowledgments}

This research was funded by National Natural Science Foundation of China (\#31160511, \#31460672) and Qinghai Science and Technology Department (\#2016ZJ-738).

6. Davies TA, Yee YC, Bush K, Sahm D, Evangelista A, Goldschmidt R. Effects of the 7-valent pneumococcal conjugate vaccine on U.S. levofloxacin-resistant Streptococcus pneumoniae. Microb Drug Resist 2008; 14: 187-196, doi: $10.1089 / \mathrm{mdr} .2008 .0805$.

7. Li L, Hassan KA, Brown MH, Paulsen IT. Rapid multiplexed phenotypic screening identifies drug resistance functions for three novel efflux pumps in Acinetobacter baumannii. J Antimicrob Chemother 2016; 71: 1223-3122, doi: 10.1093/ jac/dkv460.

8. Jarmuła A, Obłak E, Wawrzycka D, Gutowicz J. Effluxmediated antimicrobial multidrug resistance [in Polish]. Postepy Hig Med Dosw (online) 2011; 65: 216-227, doi: 10.5604/ 17322693.937011.

9. Li XZ, Nikadio H. Efflux-mediated drug resistance in bacteria: an update. Drugs 2009; 69: 1555-1623, doi: 10.2165/11317 030-000000000-00000. 
10. Bannam TL, Johanesen PA, Salvado CL, Pidot SJ, Farrow $\mathrm{KA}$, Rood Jl. The Clostridium perfringens TetA $(P)$ efflux protein contains a functional variant of the Motif $A$ region found in major facilitator superfamily transport proteins. Microbiology 2004; 150: 127-134, doi: 10.1099/mic.0.26614-0.

11. Cui L, Murakami H, Kuwahara-Arai K, Hanaki H, Hiramatsu $\mathrm{K}$. Contribution of a thickened cell wall and its glutamine nonamidated component to the vancomycin resistance expressed by Staphylococcus aureus Mu50. Antimicrob Agents Chemother 2000; 44: 2276-2285, doi: 10.1128/ AAC.44.9.2276-2285.2000.

12. Charpentier E, Tuomanen E. Mechanisms of antibiotic resistance and tolerance in Streptococcus pneumoniae. Microbes Infect 2000; 2:1855-1864, doi: 10.1016/S12864579(00)01345-9.

13. Sahal G, Bilkay IS. Multi drug resistance in strong biofilm forming clinical isolates of Staphylococcus epidermidis. Braz J Microbiol 2014; 45: 539-544, doi: 10.1590/S1517-8382 2014005000042.

14. Stewart PS. Theoretical aspects of antibiotic diffusion into microbial biofilms. Antimicrob Agents Chemother 1996; 40: 2517-2522.

15. Xu KD, Stewart PS, Xia F, Huang CT, McFeters GA. Spatial physiological heterogeneity in Pseudomonas aeruginosa biofilm is determined by oxygen availability. Appl Environ Microbiol 1998; 64: 4035-4039.

16. Ito A, Taniuchi A, May T, Kawata K, Okabe S. Increased antibiotic resistance of Escherichia coli in mature biofilms. Appl Environ Microbiol 2009; 75: 4093-4100, doi: 10.1128/ AEM.02949-08.

17. Varga JJ, Therit B, Melville SB. Type IV pili and the CcpA protein are needed for maximal biofilm formation by the gram-positive anaerobic pathogen Clostridium perfringens. Infect Immun 2008; 76: 4944-4951, doi: 10.1128/IAI.00692-08.

18. GuiSheng YE, Yong ZOU. Isolation and identification of Clostridium perfringens type $A$ in the wastewater from dining kitchen. Heilongjiang Animal Sci Vet Med 2014; 1: 103-104.

19. Li R, Yu C, Li Y, Lam TW, Yiu SM, Kristiansen K, et al. SOAP2: An improved ultrafast tool for short read alignment. Bio Informatics 2009; 25: 1966-1967, doi: 10.1093/bioinfor matics/btp336.

20. Audic S, Claverie JM. The significance of digital gene expression profiles. Genome Res 1997; 70: 986-995, doi: $10.1101 / \mathrm{gr} .7 .10 .986$.

21. Kanehisa M, Araki M, Goto S, Hattori M, Hirakawa M, Itoh M, et al. KEGG for linking genomes to life and the environment. Nucleic Acids Res 2008; 36: D480-484, doi: 10.1093/nar/ gkm882.

22. Pimenta $A C$, Fernandes $R$, Moreira IS. Evolution of drug resistance: insight on TEM $\beta$-lactamases structure and activity and $\beta$-lactam antibiotics. Mini Rev Med Chem 2014; 14: 111-122, doi: 10.2174/1389557514666140123145809.

23. Saravanan $\mathrm{M}$, Ramachandran $\mathrm{B}$, Barabadi $\mathrm{H}$. The prevalence and drug resistance pattern of extended spectrum $\beta$-lactamases (ESBLs) producing Enterobacteriaceae in Africa. Microb Pathog 2017; 114: 180-192, doi: 10.1016/j.micpath.2017. 11.061.

24. Shettigar K, Bhat DV, Satyamoorthy K, Murali TS. Severity of drug resistance and co-existence of Enterococcus faecalis in diabetic foot ulcer infections. Folia Microbiol (Praha) 2018; 63: 115-122, doi: 10.1007/s12223-017-0547-2.

25. Chang HL, Tang CH, Hsu YM, Wan L, Chang YF, Lin CT, et al. Nosocomial outbreak of infection with multidrugresistant (MDR) Acinetobacter baumannii in a medical center in Taiwan. Infect Control Hosp Epidemiol 2009; 30: 34-38, doi: 10.1086/592704.

26. Ender M, Berger-Bächi, McCallum N. A novel DNA-binding protein modulating methicillin resistance in Staphylococcus aureus. BMC Microbiol 2009; 9: 15, doi: 10.1186/14712180-9-15.

27. Pang SZ, Oberhaus SM, Rasmussen JL, Knipple DC, Bloomquist JR, Dean $\mathrm{DH}$, et al. Expression of a gene encoding a scorpion insectotoxin peptide in yeast, bacteria and plants. Gene 1992; 116: 165-172, doi: 10.1016/03781119(92)90512-N.

28. Hong IP, Lee SJ, Kim YS, Choi SG. Recombinant expression of human cathelicidin (hCAP18/LL-37) in Pichia pastoris. Biotechnol Lett 2007; 29: 73-78, doi: 10.1007/s10529-0069202-8.

29. Ngamwongsatit $B$, Tanomsridachchai $W$, Suthienkul $O$, Urairong S, Navasakuljinda W, Janvilisri T. Multidrug resistance in Clostridium perfringens isolated from diarrheal neonatal piglets in Thailand. Anaerobe 2016; 38: 88-93, doi: 10.1016/j.anaerobe.2015.12.012.

30. Catalán A, Espoz MC, Cortés W, Sagua H, González J, Araya JE. Tetracycline and penicillin resistant Clostridium perfringens isolated from the fangs and venom glands of Loxosceles laeta: its implications in loxoscelism treatment. Toxicon 2010; 56: 890-896, doi: 10.1016/j.toxicon.2010. 06.012.

31. Lyras D, Adams V, Lucet I, Rood Jl. The large resolvase TnpX is the only transposon-encoded protein required for transposition of the Tn4451/3 family of integrative mobilizable elements. Mol Microbiol 2004; 51: 1787-1800, doi: 10.1111/j.1365-2958.2003.03950.x.

32. Lyras D, Adams V, Ballard SA, Teng WL, Howarth PM, Crellin PK, et al. tISCpe8, an IS1595-family lincomycin resistance element located on a conjugative plasmid in Clostridium perfringens. J Bacteriol 2009; 191: 6345-6351, doi: 10.1128/ JB.00668-09.

33. Park M, Rooney AP, Hecht DW, Li J, McClane BA, Nayak R, et al. Phenotypic and genotypic characterization of tetracycline and minocycline resistance in Clostridium perfringens. Arch Microbiol 2010; 192: 803-810, doi: 10.1007/ s00203-010-0605-5.

34. Rafii F, Park M. Substitutions of amino acids in alpha-helix-4 of gyrase A confer fluoroquinolone resistance on Clostridium perfringens. Arch Microbiol 2007; 187: 137-144, doi: 10.1007/ s00203-006-0180-y.

35. Hölzel CS, Harms KS, Schwaiger K, Bauer J. Resistance to linezolid in a porcine Clostridium perfringens strain carrying a mutation in the rpID gene encoding the ribosomal protein L4. Antimicrob Agents Chemother 2010; 54: 1351-1353, doi: 10.1128/AAC.01208-09.

36. Rafii F, Park M, Carman RJ. Characterization of an ATPbinding cassette from Clostridium perfringens with homology to an $\mathrm{ABC}$ transporter from Clostridium hathewayi. Anaerobe 2009; 15: 116-121, doi: 10.1016/j.anaerobe.2009.01.008. 